\title{
Detachment of an Oil Droplet from Adhesive Surface Induced by Reversed Electrowetting
}

\author{
Qinggong Wang*, Ning Weng, Wei Yao \\ Qian Xuesen Laboratory of Space Technology / China Academy of Space Technology \\ No. 104, Youyi Road, Beijing, China \\ wangqinggong@qxslab.cn; wengning@qxslab.cn; yaowei@qxslab.cn
}

\section{Extended Abstract}

It is known that droplet manipulation by electrowetting (EW) has been a hot topic where the EW generally considers conductive droplets by increasing its wettability on a solid surface [1]-[3]. However, the opposite phenomenon, e.g. decreasing the wettability of a non-conductive droplet and increasing its contact angle by a reversed electrowetting (REW) effect is scarcely reported. Actuation of a non-conductive droplet and finally inducing its detachment from the adhesive surface via the REW is of the same importance in applications of microfluidics [4]. It involves not only the transient droplet dynamics but also a criteria for droplet detachment from the adhesive surface.

Comparing with the traditional EW system, the REW experimental system shows some unique features. First, in the REW setup, the needle electrode is not in contact with the target droplet, and thus the drop's motion is free both horizontally and vertically. Second, actuation of the droplet is no longer limited by the voltage of contact angle saturation in REW since the droplet's wettability on the substrate is reduced by the electric force. If the voltage is overloaded, the contact line of droplet will be excessively contracted. At some condition, the contact area will be reduced to zero, leading to the detachment of droplet from the surface. Droplet detachment occurs naturally once the applied voltage is high enough even though the friction of contact line could be significant during the actuation. Thus, the critical condition for droplet detachment in the REW should be newly established.

To uncover the details, we design an experimental setup for the REW operation and perform systematically an experimental study on the droplet detachment recently. In the experiments, silicone oil is used to generate the oil droplets, and filtered water is used as the ambient fluid. The solid substrate is made of a glass substrate coated with indium tin oxide (ITO) film and then deposited by a dielectric layer of Parylene C. Potential difference is applied between the substrate and the surrounding fluid, and the applied voltage is tuned in stepwise. The present experiments cover the full range of operation, and we identify three different regimes. An under-actuated regime occurs at low applied voltages, in which the contact angle (CA) of the droplet shows a monotonic increase with the increase of voltage $(V)$. At high voltages, the contact line of the sessile droplet is over-contracted by the REW. The droplet shows oscillation and it refers to the overactuated regime. At sufficiently high voltages, the droplet is rejected completely from the surface. The critical condition for droplet detachment is built from energy conservation. The droplet is detachable only when the excessive kinetic energy of droplet is able to overcome the adhesion work of the lyophilic surface before the energy is dissipated completely by viscous friction and liquid inertia during actuation. A critical equation for the threshold voltage of droplet detachment is built, and its validity is confirmed by the experimental results.

\section{References}

[1] M. G. Pollack, R. B. Fair, A. D. Shenderov, "Electrowetting-based actuation of liquid droplets for microfluidic applications," App. Phys. Lett., vol. 77, no. 11, pp. 1725-1726, 2000.

[2] Q. Vo, T. Tran, "Critical conditions for jumping droplets," Phys. Rev. Lett., vol. 123, no. 2, pp. 024502, 2019.

[3] M. Maillard, J. Legrand, B. Berge, "Two liquids wetting and low hysteresis electrowetting on dielectric applications," Langmuir, vol. 25, no. 11, pp. 6162-6167, 2009.

[4] Q. Wang, M. Xu, C. Wang, J. Gu, N. Hu, J. Lyu, W. Yao. "Actuation of a Non-Conductive Droplet in Aqueous Fluid by Reversed Electrowetting Effect”. Langmuir, 2020. Doi:10.1021/acs.langmuir.0c01161. 